\title{
Uniqueness of static, isotropic low-pressure solutions of the Einstein-Vlasov system
}

\author{
Maximilian Thaller ${ }^{1}$ - Tomohiro Harada ${ }^{2}$
}

Received: 9 July 2018 / Accepted: 26 February 2020 / Published online: 16 March 2020

(c) The Author(s) 2020

\begin{abstract}
In Beig and Simon (Commun Math Phys 144:373-390, 1992) the authors prove a uniqueness theorem for static solutions of the Einstein-Euler system which applies to fluid models whose equation of state fulfills certain conditions. In this article it is shown that the result of Beig and Simon (1992) can be applied to isotropic Vlasov matter if the gravitational potential well is shallow. To this end we first show how isotropic Vlasov matter can be described as a perfect fluid giving rise to a barotropic equation of state. This Vlasov equation of state is investigated, and it is shown analytically that the requirements of the uniqueness theorem are met for shallow potential wells. Finally the regime of shallow gravitational potential is investigated by numerical means. An example for a unique static solution is constructed, and it is compared to astrophysical objects like globular clusters. Finally we find numerical indications that solutions with deep potential wells are not unique.
\end{abstract}

Keywords General relativity · Vlasov matter · Perfect fluid · Uniqueness theorem

Mathematics Subject Classification $83 \mathrm{C} 20$

\section{Introduction}

In [8] Beig and Simon prove that a static solution of the Einstein-Euler system with matter quantities of compact support is spherically symmetric and uniquely determined by the surface potential of the fluid body if the matter fulfills certain assumptions. This

Maximilian Thaller

maxtha@chalmers.se

Tomohiro Harada

harada@rikkyo.ac.jp

1 Department of Mathematical Sciences, Chalmers University of Technology, 41296 Gothenburg, Sweden

2 Department of Physics, Rikkyo University, Toshima, Tokyo 171-8501, Japan 
is done by showing that a static solution of the Einstein-Euler system is isometric to a spherically symmetric solution if the assumptions of their theorem are satisfied.

This theorem thus implies two statements: First that a solutions satisfying the assumptions is spherically symmetric and second, that it is uniquely determined by the surface potential. For perfect fluids with Newtonian gravity it is in fact already known for a long time that static solutions automatically are spherically symmetric [10]. In the framework of general relativity it turned out to be surprisingly difficult to establish comparable results. An important step is the analysis of Masood-ul-Alam [12] published in 1988. A uniqueness result is given which, however, only applies to restricitive and somewhat unphysical assumptions on the equation of state. In the following years the analysis could be extended to different types of equations of state which are physically more relevant. See [11] for a chronological overview of results on symmetry and uniqueness. To our knowledge the question whether isotropic Vlasov matter, which can be seen as perfect fluid, is covered by these uniqueness results has not been addressed yet.

This article is concerned with the Einstein-Vlasov system, and it is investigated under which assumptions the main theorem of [8] can be applied to it. We have, for example, the question in mind if a globular cluster necessarily is spherically symmetric. Vlasov matter is a natural choice of matter model to describe this situation. Generally, Vlasov matter possesses features that cannot be described by a perfect fluid. In particular the momenta of the particles can be distributed anisotropically. It is known that there exist static, anisotropic solutions of the Einstein-Vlasov system which are not spherically symmetric [4]. Isotropic solutions of the static Einstein-Vlasov system, however, resemble perfect fluid solutions much more. In the non-relativistic case it is known that isotropic static solutions, so-called steady states, are necessarily spherically symmetric. This is a by-product of the method of proof of existence. It is shown that a steady state is a minimizer of the so-called energy-Casimir functional. These minimizers then turn out to be spherically symmetric. For certain choices of model parameters these solutions are, in addition, the unique steady states to a given mass. For other choices of model parameters there exist several spherically symmetric, static solutions with the same mass. See [16] for details. For the Einstein-Vlasov system similar methods have not yet been successfully applied. Some progress has been made under the assumption that the considered steady states are not very relativistic in a certain sense [9]. Existence of isotropic, spherically symmetric static solutions of the Einstein-Vlasov system has been established by other methods [14].

This picture, that not very relativistic static solutions of the Einstein-Vlasov system are necessarily spherically symmetric, whereas it is unclear for highly relativistic ones, is confirmed in this article. We show that under the assumption of isotropy the energy-momentum tensor of Vlasov matter is described by two functions $\varrho$ and $p$ which can be seen as energy density and pressure of a perfect fluid satisfying a barotropic equation of state. In terms of these functions $\varrho$ and $p$, an additional function $I$, defined in (2.3) below, can be introduced. By the uniqueness theorem of [8] a static fluid solution is unique if $I \leq 0$. So we analyze the equation of state resulting from isotropic Vlasov matter and show analytically that $I \leq 0$ in the regime of relatively low pressure. The applied method is very robust so that the effect of different choices of ansatz functions for the particle distribution can be studied. We are able to 
characterize a large class of solutions which will be unique in the low-pressure regime. At the same time we are able to give criteria on the particle distribution function revealing that the resulting equation of state is not compatible with the uniqueness result of [8].

In the last part of the paper the low-pressure regime is investigated further by numerical means. We calculate explicit examples of static solutions of the EinsteinVlasov system in spherical symmetry with low pressures. Due to the analytical result of this article we conclude that these solutions are unique. Further, we discuss numerical indications that in the high-pressure regime static solutions are not unique; namely, we find different spherically symmetric solutions which have the same surface potential.

For an isotropic particle distribution function of the Vlasov matter, the maximum pressure and the concentration parameter $\Gamma$ of a spherically symmetric solution are correlated. Therefore, static solutions of the Einstein-Vlasov system with a lowconcentration parameter $\Gamma$ are necessarily spherically symmetric. We calculate the maximum concentration parameter in the low-pressure regime for an example family of particle distribution functions and set this into relation to observational values of existing astrophysical objects. At the example of the numerically calculated family of solutions we will see that solutions with a concentration parameter comparable to neutron stars are not in the low-pressure regime and the main theorem of [8] cannot be applied. Stars or globular clusters however are in this regime.

\section{Preliminaries}

\subsection{Fluid models}

In this article units in which $G=c=1$ are used. Moreover, we use the Einstein summation convention. Greek indices run from 0 to 3 , and Latin indices run from 1 to 3 . Let $\mathscr{M}$ be a four-dimensional manifold equipped with a Lorentzian metric $g$. We assume that $(\mathscr{M}, g)$ is a static space-time. This means that there exists a threedimensional manifold $\Sigma$ such that $\mathscr{M} \cong \mathbb{R} \times \Sigma$ and there exist coordinates $t, x^{1}, x^{2}$, $x^{3}$ such that the metric $g$ can be written as

$$
g=-V^{2}\left(x^{1}, x^{2}, x^{3}\right) \mathrm{d} t^{2}+\gamma_{a b}\left(x^{1}, x^{2}, x^{3}\right) \mathrm{d} x^{a} \mathrm{~d} x^{b}
$$

where $V \in C^{1}(\Sigma ; \mathbb{R})$ and the Riemannian metric $\gamma_{a b}$ is the restriction of $g$ to $\Sigma$.

Assume that on $\Sigma$ we have two functions $\varrho, p \in C^{2}(\Sigma ; \mathbb{R})$. Assume furthermore that the support of these functions is compact and let $Q \subset \Sigma$ be an open set such that $\bar{Q}=\operatorname{supp}(\varrho) \cup \operatorname{supp}(p)$. If the functions $\varrho$ and $p$ are related by a barotropic equation of state, i.e., $\varrho=\varrho(p), \mathrm{d} \varrho / \mathrm{d} p \geq 0$, then they give rise to a fluid model which we define as in [8].

Definition 1 (Fluid model) Let $\varrho, p \in C^{2}(\Sigma ; \mathbb{R})$ satisfy a barotropic equation of state, i.e., $\varrho=\varrho(p), \mathrm{d} \varrho / \mathrm{d} p \geq 0$. A corresponding fluid model is a triple $\left(\Sigma, \gamma_{a b}, V\right)$, where $\Sigma$ is a three-dimensional Riemannian manifold endowed with the metric $\gamma_{a b}$, and $V \in C^{1}(\Sigma ; \mathbb{R})$ such that the Einstein equations 


$$
\begin{aligned}
R_{a b} & =\frac{1}{V} D_{a} D_{b} V+4 \pi(\varrho-p) g_{a b}, \\
\Delta V & =4 \pi V(\varrho+3 p),
\end{aligned}
$$

hold. Here $D_{a}$ is the covariant derivative formed from $\gamma_{a b}, \Delta=\gamma^{a b} D_{a} D_{b}$, and $R_{a b}$ is the Ricci tensor formed from $\gamma_{a b}$.

Next we define the quantity

$$
I=\frac{1}{5} \kappa^{2}+2 \kappa+(\varrho+p) \frac{\mathrm{d} \kappa}{\mathrm{d} p}, \quad \text { where } \quad \kappa=\frac{\varrho+p}{\varrho+3 p} \frac{\mathrm{d} \varrho}{\mathrm{d} p} .
$$

We review the main theorem of [8] that this article relies on.

Theorem 1 (Beig \& Simon 1990) Assume we are given a static perfect fluid model $\left(\Sigma, \gamma_{a b}, V\right)$ with equation of state satisfying $I \leq 0$, and a spherically symmetric solution $\left(\mathbb{R}^{3},{ }^{0} \gamma_{a b},{ }^{0} V\right)$. Then, the given model and the spherically symmetric solution are isometric.

The function ${ }^{0} V$ turns, by the theorem, out to be the same as $V$, and it depends on the radial coordinate only. Moreover, it is monotonically increasing. So the theorem is a uniqueness result in the sense that the value of $V$ at the boundary $\partial Q$ of the fluid body uniquely determines the space-time (for a fixed equation of state). The statement that all static solutions, which have an equation of state such that the assumptions of the theorem are satisfied, are spherically symmetric is an immediate consequence. We call this value of $V$ the surface potential. Later in the analysis (Sect. 4), a cutoff energy $E_{0}$ will be introduced. Its value will be exactly this surface potential.

\subsection{Vlasov matter}

We consider an ensemble of particles in $\mathscr{M}$ which move along timelike geodesics. Let $x(\sigma)=\left(x^{0}(\sigma), x^{1}(\sigma), x^{2}(\sigma), x^{3}(\sigma)\right)$ be a future-directed geodesic and let

$$
p^{\mu}(\sigma):=\frac{\mathrm{d}}{\mathrm{d} \sigma} x^{\mu}(\sigma)
$$

be the canonical momenta. Then, $p^{\mu}(\sigma)$ fulfills the geodesic equation

$$
\frac{\mathrm{d} p^{\mu}}{\mathrm{d} \sigma}-\Gamma_{\nu \lambda}^{\mu} p^{v} p^{\lambda}=0, \quad \mu=0, \ldots, 3
$$

The rest mass $m$ of the particle following the geodesic $x^{\mu}(\sigma)$ is defined by

$$
m^{2}=-g_{\mu \nu}(x(\sigma)) p^{\mu}(\sigma) p^{v}(\sigma) .
$$

It can be shown [18] that the rest mass $m$ stays constant along the geodesic $x^{\mu}(\sigma)$. We note that the parameter $\sigma$ is proper time if and only if $m=1$. Otherwise, we have $d \sigma=d \tau / m$ so that $p^{\mu}=d x^{\mu} / d \sigma$. 
The mass shell $P_{m}$ is defined to be

$$
P_{m}=\left\{(x, p) \in T \mathscr{M}: g_{\mu v}(x) p^{\mu} p^{v}=-m^{2}, p \text { future directed }\right\}
$$

The mass shell is a seven-dimensional submanifold of $T \mathscr{M}$ containing the lifts, to the tangent bundle $T \mathscr{M}$, of the future-directed geodesics in $\mathscr{M}$, corresponding to particles with rest mass $m$. On $P_{m}$ we define the distribution function $f \in C^{1}\left(P_{m} ; \mathbb{R}\right)$ of the particles with rest mass $m$ which satisfies the Vlasov equation,

$$
p^{\mu} \frac{\partial}{\partial x^{\mu}} f-\Gamma_{\nu \lambda}^{i} p^{\nu} p^{\lambda} \frac{\partial}{\partial p^{i}} f=0 .
$$

We will write $\left(p^{1}, p^{2}, p^{3}\right)$ as $\mathbf{p}$ and $\left(x^{1}, x^{2}, x^{3}\right)$ as $\mathbf{x}$ etc. The stress-energy tensor for $m>0$ is given by

$$
T^{\mu \nu}\left(x^{\sigma}\right)=\frac{1}{m} \int_{P_{(m, x)}} f\left(x^{\sigma}, \mathbf{p}\right) p^{\mu} p^{v} \mu_{P_{(m, x)}},
$$

where $P_{(m, x)}$ is the fiber of the mass shell $P_{m}$ which is a submanifold of $T_{x} \mathscr{M}$, and $\mu_{P_{(m, x)}}$ is the volume form on $P_{(m, x)}$. For the massless case, $m=0$, a formula can be obtained by a continuity argument, cf. (3.10) below. We should note that $T^{\mu \nu}$ behaves as a covariant tensor in spite of the apparently three-dimensional volume integral. We also define the particle number current as

$$
N^{\mu}\left(x^{\sigma}\right)=\frac{1}{m} \int_{P_{(m, x)}} f\left(x^{\sigma}, \mathbf{p}\right) p^{\mu} \mu_{P_{(m, x)}} .
$$

One can show the conservation laws for the above quantities, $\nabla_{\mu} T^{\mu \nu}=0, v=$ $0, \ldots, 3$, and $\nabla_{\mu} N^{\mu}=0$. See [1] for a review article on the Einstein-Vlasov system and [18] for more details on the geometric setup.

\section{Tetrad description}

A solution $(\mathscr{M}, g, f)$ of the Einstein-Vlasov system is a Lorentzian metric $g$, defined on the manifold $\mathscr{M}$, such that the Einstein equations, $G_{\mu \nu}=8 \pi T_{\mu \nu}, \mu, \nu=0, \ldots, 3$, are satisfied where the Einstein tensor $G_{\mu \nu}$ is calculated from the metric $g$. Further, $f$ is a particle distribution function satisfying the Vlasov equation (2.8) and giving via (2.9) rise to the energy-momentum tensor $T_{\mu} \nu$ on the right hand side of the Einstein equations.

In this section we show that a solution of the Einstein-Vlasov system with isotropic particle distribution function is a fluid model in the sense of Definition 1. To this end we first show in Lemma 1 how to express the energy-momentum tensor (2.9) of Vlasov matter in the form of a perfect fluid. In this section, by $\eta^{A B}$ we denote the components of the Minkowski metric, i.e., $\eta^{00}=-1, \eta^{0 I}=\eta^{I 0}=0$ and $\eta^{I J}=\delta^{I J}$ for $I, J=1,2,3$. 
The tangent bundle $T \mathscr{M}$ of $\mathscr{M}$ can be seen as eight-dimensional manifold which is naturally equipped with the coordinates $x^{\mu}, p^{\nu}, \mu, v=0, \ldots, 3$, where $p^{\mu}$ is the canonical momentum corresponding to the coordinate $x^{\mu}$. However, to formulate isotropic distribution, it is useful to introduce an orthonormal basis $\left\{e_{(A)}\right\}$ $(A=0,1,2,3)$ for the tangent bundle, i.e., $g\left(e_{(A)}, e_{(B)}\right)=\eta_{A B}$, which we call a tetrad basis. We define $v^{(A)}$ as the components of the vector $p^{\mu} \partial_{x^{\mu}}$ with respect to this tetrad frame $\left\{e_{(A)}\right\}$ so that $p^{\mu} \partial_{x^{\mu}}=v^{(A)} e_{(A)}$, where and hereafter we adopt Einstein convention for summation with respect also to the tetrad components with $A, B=0,1,2,3$ and $I, J, K, L=1,2,3$. Let $e_{(A)}^{\mu}$ be the coefficients of this frame, i.e., $e_{(A)}=e_{(A)}^{\mu} \partial_{x^{\mu}}$. Then, we have the identity

$$
g^{\mu \nu}=\eta^{A B} e_{(A)}^{\mu} e_{(B)}^{\nu} .
$$

We use the notation $x:=\left(x^{0}, x^{1}, x^{2}, x^{3}\right)$ and $\mathbf{v}:=\left(v^{(1)}, v^{(2)}, v^{(3)}\right)$ for abbreviation.

Definition 2 A matter distribution function $f \in C^{1}\left(P_{m} ; \mathbb{R}\right)$ is called isotropic if there exist a tetrad basis $\left\{e_{(A)}\right\}(A=0,1,2,3)$ and a function $F: \mathbb{R}^{4} \times \mathbb{R}_{+} \rightarrow \mathbb{R}_{+}$such that

$$
f(x, \mathbf{p})=F(x, v), \quad v^{2}=\delta_{I J} v^{(I)} v^{(J)},
$$

for all $(x, \mathbf{v}) \in P_{m}$. We call $F$ an isotropic ansatz function.

Proposition 1 Let $(\mathscr{M}, g)$ be a four-dimensional Lorentzian space-time and $P_{m}$ the corresponding mass shell for $m \geq 0$, equipped with the coordinates $x$ and $\mathbf{p}$ as described above. Further, let $F: \mathbb{R}^{4} \times \mathbb{R}_{+} \rightarrow \mathbb{R}_{+}$be an isotropic ansatz function for the matter distribution function $f$, satisfying

$$
F(x, v)=\mathcal{O}\left(v^{-4-\epsilon}\right), \quad \epsilon>0
$$

for $v \in \mathbb{R}_{+}$. Further let

$$
\begin{aligned}
& \varrho(x):=4 \pi \int_{0}^{\infty} F(x, v) v^{2} \sqrt{m^{2}+v^{2}} \mathrm{~d} v, \\
& p(x):=\frac{4 \pi}{3} \int_{0}^{\infty} F(x, v) \frac{v^{4}}{\sqrt{m^{2}+v^{2}}} \mathrm{~d} v .
\end{aligned}
$$

Then, there exists a unit timelike vector field $u$ such that the energy-momentum tensor $T^{\mu \nu}$ defined in (2.9) takes the form

$$
T^{\mu \nu}=\varrho u^{\mu} u^{v}+p\left(u^{\mu} u^{v}+g^{\mu v}\right) .
$$

Proof First we express the components $T^{\mu \nu}(x)$ of the energy-momentum tensor, defined in (2.9), in terms of $\mathbf{v}$ in the tangent space $T_{x} \mathscr{M}$. To this end we calculate 
the volume form $\mu_{P_{(m, x)}}$ of the fiber $P_{(m, x)}$ which is a submanifold of $T_{x} \mathscr{M}$. Note that the mass shell condition reads

$$
v^{(0)}=\sqrt{m^{2}+v^{2}}
$$

The tangent space $T_{x} \mathscr{M}$ can be seen as a four-dimensional manifold endowed with the Minkowski metric in the coordinates $v^{(A)}, A=0,1,2,3$. For $m>0$, using (3.7) we calculate the restriction $\rho$ of the Minkowski metric to $P_{(m, x)}$. We obtain

$$
\rho=\delta_{I J} \mathrm{~d} v^{(I)} \mathrm{d} v^{(J)}-\delta_{I K} \delta_{J L} \frac{v^{(I)} v^{(J)}}{\left(v^{(0)}\right)^{2}} \mathrm{~d} v^{(K)} \mathrm{d} v^{(L)} .
$$

Thus, we have

$$
\mu_{P_{(m, x)}}=\sqrt{|\operatorname{det}(\rho)|} \mathrm{d} v^{(1)} \mathrm{d} v^{(2)} \mathrm{d} v^{(3)}=\frac{m}{v^{(0)}} \mathrm{d} v^{(1)} \mathrm{d} v^{(2)} \mathrm{d} v^{(3)} .
$$

This formula is valid in the massive case. In the massless case, however, the metric (3.9) is degenerate. Since $p^{\mu}=e_{(A)}^{\mu} v^{(A)}$, a straightforward calculation using (3.1) yields the formula

$$
T^{\mu \nu}(x)=\left.\left.e_{(A)}^{\mu}\right|_{x} e_{(B)}^{\nu}\right|_{x} \int_{\mathbb{R}^{3}} f(x, v) v^{(A)} v^{(B)} \frac{1}{v^{(0)}} \mathrm{d} v^{(1)} \mathrm{d} v^{(2)} \mathrm{d} v^{(3)}
$$

for the energy-momentum tensor. The notation $\left.e_{(A)}^{\mu}\right|_{x}$ denotes that the vector $e_{(A)}^{\mu}$ is evaluated at the space-time point $x$. By a continuity argument this formula is also valid in the massless case, $m=0$. Then, the formulas (3.4) and (3.5) for $\varrho$ and $p$ yield

$$
T^{\mu \nu}(x)=\left.\left.e_{(0)}^{\mu}\right|_{x} e_{(0)}^{v}\right|_{x} \varrho(x)+\left(\left.\left.e_{(0)}^{\mu}\right|_{x} e_{(0)}^{\nu}\right|_{x}+g^{\mu \nu}(x)\right) p(x)
$$

Finally we check that

$$
u(x):=\left.e_{(0)}\right|_{x}=\left.e_{(0)}^{\mu}\right|_{x} \partial_{x}^{\mu}
$$

is a timelike unit vector field. Using $g\left(e_{(0)}, e_{(0)}\right)=-1$ one establishes all desired properties of $u$ and the lemma is shown.

For the static case, we can identify $u=e_{(0)}=e_{(0)}^{0} \partial_{t}$ and write $F(x, v)=F(\mathbf{x}, v)$, where $\mathbf{x}:=\left(x^{1}, x^{2}, x^{3}\right)$. Taking an energy-momentum tensor of the form (3.6) as right hand side to Einstein's equations $G_{\mu \nu}=8 \pi T_{\mu \nu}$ and calculating the Einstein tensor $G_{\mu \nu}$ on the left hand side from a metric of the form (2.1) one finds the system (2.2a)-(2.2b) in Definition 1 of a fluid model.

By inspection of the formulas (4.4) and (4.5) below one notices that both $\varrho$ and $p$ are decreasing with respect to $V$. Thus, $p(V)$ can be inverted and we write $V(p)=$ $p^{-1}(p)$. Further, $\varrho$ and $p$ fulfill a barotropic equations of state. By slight abuse of notation we write $\varrho(p)=\varrho(V(p))$. In conclusion, in this section we have seen that 
an isotropic static solution of the Einstein-Vlasov system is a fluid model in the sense of Definition 1.

\section{Main result}

In this section we set $m=1$ and we assume that the metric $g$ is of the form (2.1). Since $(\mathscr{M}, g)$ is a static space-time by assumption, the timelike vector field $\partial_{t}$ is Killing. Then, the quantity

$$
E=-g\left(p^{\mu}, \partial_{t}\right)=V(\mathbf{x})^{2} p^{0}=V(\mathbf{x}) \sqrt{1+v^{2}}, \quad \mathbf{x} \in \mathbb{R}^{3}
$$

is conserved along the geodesics with tangent vector $p^{\mu}$. Note that the mass shell condition (2.7) and the frame components have been used in the formula (4.1) for the particle energy $E$, and recall that we denote $v^{2}=\delta_{I J} v^{(I)} v^{(J)}$. This implies that if $f$ depends on $x$ and $v$ only indirectly via $E$, it satisfies the Vlasov equation. Henceforth, we assume that $f$ is a function of $E$. We denote this function by $\Phi$. Moreover, we assume that there exists a cutoff energy $E_{0}>0$. This means that it is assumed that no particle has energy $E$ larger than this value. In other words $\Phi(E)=0$ if $E>E_{0}$. For technical reasons we introduce the function $\phi:(-\infty, 1] \rightarrow \mathbb{R}$, vanishing on $(-\infty, 0)$, so that we can write

$$
F(\mathbf{x}, v)=\Phi(E(\mathbf{x}, v))=: \phi\left(1-\frac{E(\mathbf{x}, v)}{E_{0}}\right)
$$

Since $E$ depends only on the absolute value $v$ of $\mathbf{v}, \Phi$ is an isotropic particle distribution function, cf. Definition 2.

From a change of variables in the integrals (3.4) and (3.5), given by

$$
v=\sqrt{\left(\frac{E}{V}\right)^{2}-1}, \quad d v=\frac{\frac{E}{V}}{\sqrt{\left(\frac{E}{V}\right)^{2}-1}} \frac{\mathrm{d} E}{V}
$$

we find the formulas

$$
\begin{aligned}
& \rho=4 \pi \frac{1}{V^{3}} \int_{V}^{E_{0}} \phi\left(1-\frac{E}{E_{0}}\right) E^{2} \sqrt{\left(\frac{E}{V}\right)^{2}-1} \mathrm{~d} E, \\
& p=\frac{4 \pi}{3} \frac{1}{V} \int_{V}^{E_{0}} \phi\left(1-\frac{E}{E_{0}}\right)\left[\left(\frac{E}{V}\right)^{2}-1\right]^{3 / 2} \mathrm{~d} E .
\end{aligned}
$$

Since $\varrho(V)=p(V)=0$ for all $V \geq E_{0}$, we call $E_{0}$ the surface potential.

The results presented in this paragraph hold for ansatz functions $\phi$ that satisfy the following assumptions. We assume that $\phi:(-\infty, 1] \rightarrow \mathbb{R}_{+}$is an analytic function on $[0,1], \phi(x)=0$ if $x<0$, and that 


$$
\exists n \in \mathbb{N}: \quad \phi^{\prime}(0)=\cdots=\phi^{(n-1)}(0)=0, \quad \phi^{(n)}(0)>0 .
$$

In particular this implies that the $n$-th derivative is discontinuous at 0 . The step function $\phi(x)=\chi_{[0,1]}(x)$ or functions like $\phi(x)=[x]_{+}$and $e^{x} \chi_{[0,1]}(x)$ meet these requirements. Now we are ready to state the main result.

Theorem 2 Let $E_{0}>0$ and let $\phi$ be an ansatz function satisfying the assumption (4.6) with $n \leq 3$. Let $\left(\Sigma, \gamma_{a b}, V\right)$ be the fluid model corresponding to $\varrho$ and $p$ constructed from $\phi$ via (4.4) and (4.5). Then, there exists $p_{0}>0$ such that if $\sup _{x \in \Sigma} p(x) \leq p_{0}$, the model is spherically symmetric and the unique solution of the Einstein-Vlasov system determined by $\phi$ and the surface potential $E_{0}$.

Remark 1 It turns out that the condition $n \leq 3$ is necessary if we use ansatz functions of the form (4.6). If $n \geq 4$, so in particular the choice $\phi(x)=\left[x^{4}\right]+$ will lead to $I(p) \rightarrow \infty$, as $p \rightarrow 0$ and the main Theorem of [8] cannot be applied, i.e., it neither can be deduced that the solution is unique nor that it is not unique.

Remark 2 The condition that the ansatz function $\Phi(E)=\phi\left(1-E / E_{0}\right)$ must not be "too smooth" at $E=E_{0}$ seems to be necessary to admit static solutions with finite mass and matter quantities of compact support. In the case of polytropes, i.e. $\Phi(E)=$ $(1-E / E 0)^{k}$ there holds that if $k>7 / 2$ then there exist no corresponding solutions with finite radius and finite mass, i.e. no solution that Theorem 2 would apply to. In [7, Theorem 5.5] this is proven rigorously for the Vlasov-Poisson system, and in [17] this is done for the Einstein-Vlasov System (cf. third formula on page 3). Moreover, in [17] the authors state that whether or not the solution has finite mass and finite radius is determined by a "local condition", i.e. the behavior of $\Phi(E)$ close to $E=E_{0}$ (cf. the abstract of [17]), as it is the case in the present context.

In the proof of Theorem 2 the functions defined in (4.4) and (4.5) play an important role. For this reason we first establish some technical lemmas to treat these functions, before we state the proof of Theorem 2. It is convenient to introduce for $\kappa \in\left\{\frac{3}{2}, \frac{1}{2},-\frac{1}{2}\right\}$ the functions

$$
\xi_{\kappa}(V)=\frac{4 \pi}{V} \int_{V}^{E_{0}} \phi\left(1-\frac{E}{E_{0}}\right)\left(\frac{E^{2}}{V^{2}}-1\right)^{\kappa} \mathrm{d} E, \quad V \in\left(0, E_{0}\right] .
$$

Observe that

$$
\varrho(V)=\xi_{\frac{3}{2}}(V)+\xi_{\frac{1}{2}}(V), \quad \text { and } \quad p(V)=\frac{1}{3} \xi_{\frac{3}{2}}(V)
$$

if $V \in\left(0, E_{0}\right]$, whereas $\varrho(V)=p(V)=0$ if $V>E_{0}$. We collect some facts about the functions $\xi_{\kappa}$ in the following lemmas.

Lemma 1 Let $\phi \in H^{1}\left((-\infty, 1] ; \mathbb{R}_{+}\right)$. Then,

$$
\frac{\xi_{\kappa+1}(V)}{\xi_{\kappa}(V)} \rightarrow 0, \text { as } V \rightarrow E_{0}
$$


Furthermore, if $\phi$ satisfies (4.6), there exists $0<V^{*}<E_{0}$ such that for all $V \in$ $\left[V^{*}, E_{0}\right]$ we have

$$
\frac{\xi_{\frac{1}{2}}(V)}{\xi_{-\frac{1}{2}}(V)} \leq \frac{101\left(E_{0}+V\right)\left(E_{0}-V\right)}{100(3+2 n) V^{2}}
$$

where $n$ is introduced in (4.6).

Proof We have

$$
\xi_{\kappa+1}(V)=\frac{4 \pi}{V} \int_{V}^{E_{0}} \Phi(E)\left(\frac{E^{2}}{V^{2}}-1\right)^{\kappa+1} \mathrm{~d} E \leq\left(\frac{E_{0}^{2}}{V^{2}}-1\right) \xi_{\kappa}(V) .
$$

So

$$
\frac{\xi_{\kappa+1}(V)}{\xi_{\kappa}(V)} \leq \frac{E_{0}^{2}-V^{2}}{V^{2}}
$$

The right hand side clearly goes to zero as $V \rightarrow E_{0}$, and the first claim (4.9) of the lemma is shown.

For the proof of the second claim (4.10) we first note

$$
\begin{gathered}
\xi_{\frac{1}{2}}(V) \leq \frac{4 \pi \sqrt{E_{0}+V}}{V^{2}} \int_{V}^{E_{0}} \phi\left(\frac{E_{0}-E}{E_{0}}\right) \sqrt{E-V} \mathrm{~d} E, \\
\xi_{-\frac{1}{2}}(V) \geq \frac{4 \pi}{\sqrt{E_{0}+V}} \int_{V}^{E_{0}} \phi\left(\frac{E_{0}-E}{E_{0}}\right) \frac{1}{\sqrt{E-V}} \mathrm{~d} E
\end{gathered}
$$

since $E \leq E_{0}$. Then, we define

$$
\epsilon:=E_{0}-V
$$

and perform a change of variables in the integrals of $\xi_{\frac{1}{2}}$ and $\xi_{-\frac{1}{2}}$, given by

$$
\begin{aligned}
y & =E-V, \quad \Leftrightarrow \quad E=y+V, \\
\mathrm{~d} E & =\mathrm{d} y, \\
y(V) & =0, \quad y\left(E_{0}\right)=\epsilon .
\end{aligned}
$$

This yields

$$
\begin{aligned}
\xi_{\frac{1}{2}}(V) & \leq \frac{4 \pi \sqrt{E_{0}+V}}{V^{2}} \int_{0}^{\epsilon} \phi\left(\frac{\epsilon-y}{E_{0}}\right) \sqrt{y} \mathrm{~d} y, \\
\xi_{-\frac{1}{2}}(V) & \geq \frac{4 \pi}{\sqrt{E_{0}+V}} \int_{0}^{\epsilon} \phi\left(\frac{\epsilon-y}{E_{0}}\right) \frac{1}{\sqrt{y}} \mathrm{~d} y .
\end{aligned}
$$


We consider

$$
\begin{gathered}
\frac{V^{2}}{4 \pi \sqrt{E_{0}+V}} \xi_{\frac{1}{2}}(V)-\frac{\sqrt{E_{0}+V}}{4 \pi} X \epsilon \xi_{-\frac{1}{2}}(V) \\
\leq \int_{0}^{\epsilon} \phi\left(\frac{\epsilon-y}{E_{0}}\right)\left(\sqrt{y}-X \epsilon \frac{1}{\sqrt{y}}\right) \mathrm{d} y,
\end{gathered}
$$

where we later will substitute

$$
X=\frac{101}{100} \frac{1}{3+2 n}
$$

Recall that we assume that $\phi$ is analytic on $[0,1]$, and fulfills (4.6). Then we can write for all $x \in[0,1]$

$$
\phi(x)=\frac{\phi^{(n)}(0)}{n !} x^{n}+\frac{\phi^{(n+1)}\left(z_{x}\right)}{(n+1) !} x^{n+1},
$$

where $z_{x} \in[0,1]$ is a number depending on $x$. Now the integral in (4.19) can be calculated explicitly. This yields

$$
\begin{aligned}
& \frac{V^{2}}{4 \pi \sqrt{E_{0}+V}} \xi_{\frac{1}{2}}(V)-\frac{\sqrt{E_{0}+V}}{4 \pi} X \epsilon \xi_{-\frac{1}{2}}(V) \\
& \leq \frac{\phi^{(n)}(0)}{n ! E_{0}^{n}} \epsilon^{n+\frac{3}{2}}\left(\sum_{i=0}^{n}\left(\begin{array}{l}
n \\
i
\end{array}\right) \frac{(-1)^{i}}{i+\frac{3}{2}}-X \sum_{j=0}^{n}\left(\begin{array}{c}
n \\
j
\end{array}\right) \frac{(-1)^{j}}{j+\frac{1}{2}}\right) \\
& +\frac{\left\|\phi^{(n+1)}\right\|_{L^{\infty}([0,1])} \epsilon^{n+\frac{5}{2}}}{(n+1) ! E_{0}^{n+1}} \\
& \left|\sum_{k=0}^{n+1}\left(\begin{array}{l}
n+1 \\
k
\end{array}\right) \frac{(-1)^{k}}{k+\frac{3}{2}}-X \sum_{\ell=0}^{n+1}\left(\begin{array}{l}
n+1 \\
\ell
\end{array}\right) \frac{(-1)^{\ell}}{\ell+\frac{1}{2}}\right|
\end{aligned}
$$

We observe that the first term will be dominating for $\epsilon$ sufficiently small. By Lemma 2 below and the choice (4.20) for $X$ the first term is negative. Thus,

$$
\begin{aligned}
& \frac{V^{2}}{4 \pi \sqrt{E_{0}+V}} \xi_{\frac{1}{2}}(V)-\frac{\sqrt{E_{0}+V}}{4 \pi} X \epsilon \xi_{-\frac{1}{2}}(V) \leq 0 \\
& \Leftrightarrow \quad \frac{\xi_{\frac{1}{2}}(V)}{\xi_{-\frac{1}{2}}(V)} \leq \frac{101\left(E_{0}+V\right) \epsilon}{100(3+2 n) V^{2}}
\end{aligned}
$$

and the second claim (4.10) is established. 
Lemma 2 Let $n \geq 0$. Then, we have

$$
\sum_{i=0}^{n}\left(\begin{array}{l}
n \\
i
\end{array}\right) \frac{(-1)^{i}}{i+\frac{3}{2}}=\frac{1}{2 n+3} \sum_{j=0}^{n}\left(\begin{array}{l}
n \\
j
\end{array}\right) \frac{(-1)^{j}}{j+\frac{1}{2}}
$$

Proof We notice that the sums are given by the hypergeometric functions,

$$
\begin{aligned}
& { }_{2} F_{1}\left(-n, \frac{3}{2} ; \frac{5}{2} ; z\right)=\frac{3}{2} \sum_{k=0}^{n}\left(\begin{array}{l}
n \\
k
\end{array}\right) \frac{(-1)^{k}}{k+\frac{3}{2}} z^{k}, \\
& { }_{2} F_{1}\left(-n, \frac{1}{2} ; \frac{3}{2} ; z\right)=\frac{1}{2} \sum_{k=0}^{n}\left(\begin{array}{l}
n \\
k
\end{array}\right) \frac{(-1)^{k}}{k+\frac{1}{2}} z^{k},
\end{aligned}
$$

evaluated at $z=1$. For the values of these functions we have by the $\mathrm{Chu}-$ Vandermonde identity

$$
\begin{aligned}
& { }_{2} F_{1}\left(-n, \frac{3}{2} ; \frac{5}{2} ; 1\right)=\frac{n !}{\frac{5}{2} \cdots \frac{2 n+1}{2} \frac{2 n+3}{2}}, \\
& { }_{2} F_{1}\left(-n, \frac{1}{2} ; \frac{3}{2} ; 1\right)=\frac{n !}{\frac{3}{2} \frac{5}{2} \cdots \frac{2 n+1}{2}} .
\end{aligned}
$$

The assertion now follows.

Lemma 3 Let $E_{0}>0$ and $\Phi \in H^{1}\left(\left[0, E_{0}\right] ; \mathbb{R}\right)$. Then, the function $\xi_{\kappa}$, defined in (4.7), is continuously differentiable for $\kappa \in\left\{\frac{3}{2}, \frac{1}{2}\right\}$ and we have

$$
\begin{aligned}
& \xi_{\frac{3}{2}}^{\prime}(V)=-\frac{1}{V}\left(4 \xi_{\frac{3}{2}}(V)+3 \xi_{\frac{1}{2}}(V)\right), \\
& \xi_{\frac{1}{2}}^{\prime}(V)=-\frac{1}{V}\left(2 \xi_{\frac{1}{2}}(V)+\xi_{-\frac{1}{2}}(V)\right) .
\end{aligned}
$$

Proof Let $\Delta>0$ small. We consider

$$
\begin{aligned}
\frac{1}{\Delta} & {\left[\int_{V-\Delta}^{E_{0}} \Phi(E)\left(\frac{E^{2}}{(V-\Delta)^{2}}-1\right)^{\kappa} \mathrm{d} E-\int_{V}^{E_{0}} \Phi(E)\left(\frac{E^{2}}{V^{2}}-1\right)^{\kappa} \mathrm{d} E\right] } \\
= & \frac{1}{\Delta} \int_{V-\Delta}^{V} \Phi(E)\left(\frac{E^{2}}{(V-\Delta)^{2}}-1\right)^{\kappa} \mathrm{d} E \\
& +\int_{V}^{E_{0}} \Phi(E) \frac{1}{\Delta}\left[\left(\frac{E^{2}}{(V-\Delta)^{2}}-1\right)^{\kappa}-\left(\frac{E^{2}}{V^{2}}-1\right)^{\kappa}\right] \mathrm{d} E .
\end{aligned}
$$

If $\kappa>0$, then the first integral on the right hand side of (4.33) goes to zero, as $\Delta \rightarrow 0$. So the derivative of the integral can be obtained by merely differentiating the integrand with respect to $V$. 
The arguments in the proof of Lemma 3 cannot be applied to $\xi_{-\frac{1}{2}}$ since the first summand in (4.33) does not converge to 0 as $\Delta \rightarrow 0$ for $\kappa=-\frac{1}{2}$. Thus, the analysis of the derivative of $\xi_{-\frac{1}{2}}$ requires a different approach. The derivative $\frac{\mathrm{d}}{\mathrm{d} V} \xi_{-\frac{1}{2}}(V)$ consists in two parts,

$$
\frac{\mathrm{d}}{\mathrm{d} V} \xi_{-\frac{1}{2}}(V)=-\frac{1}{V}\left(\xi_{-\frac{1}{2}}(V)+\zeta(V)\right)
$$

where

$$
\zeta(V):=-4 \pi \frac{\mathrm{d}}{\mathrm{d} V}\left(\int_{V}^{E_{0}} \Phi(E)\left(\frac{E^{2}}{V^{2}}-1\right)^{-\frac{1}{2}} \mathrm{~d} E\right)
$$

We proof the following lemma.

Lemma 4 If $n \leq 3$ ( $n$ is introduced in the assumption (4.6) on the ansatz function $\phi$ ), then for $\epsilon>0$, (defined in 4.15) sufficiently small, we have

$$
\left|\frac{\xi_{\frac{1}{2}}}{\xi_{-\frac{1}{2}}} \frac{\zeta}{\xi_{-\frac{1}{2}}}\right|<\frac{4}{5}
$$

Proof The first factor is already treated in Lemma 1. So we focus on the second factor. First we calculate $\zeta(V)$. To this end we perform a change of variables in the integral (4.35), given by

$$
\begin{aligned}
x & =\frac{E-V}{E_{0}-V}, \quad \Leftrightarrow \quad E=x\left(E_{0}-V\right)+V, \\
\mathrm{~d} E & =\left(E_{0}-V\right) \mathrm{d} x, \\
x(V) & =0, \quad x\left(E_{0}\right)=1 .
\end{aligned}
$$

This yields

$$
\begin{aligned}
\zeta(V)= & -4 \pi \frac{\mathrm{d}}{\mathrm{d} V}\left(\left(E_{0}-V\right) \int_{0}^{1} \phi\left(\frac{\left(E_{0}-V\right)(1-x)}{E_{0}}\right)\right. \\
& \left.\times\left(\left(x\left(\frac{E_{0}}{V}-1\right)+1\right)^{2}-1\right)^{-\frac{1}{2}} \mathrm{~d} x\right) .
\end{aligned}
$$

A straightforward calculation yields

$$
\zeta(V)=\zeta_{1}(V)+\zeta_{2}(V),
$$


where

$$
\begin{aligned}
& \zeta_{1}(V)=4 \pi \int_{V}^{E_{0}} \phi^{\prime}\left(1-\frac{E}{E_{0}}\right) \frac{E_{0}-E}{E_{0}\left(E_{0}-V\right)}\left(\frac{E^{2}}{V^{2}}-1\right)^{-\frac{1}{2}} \mathrm{~d} E \\
& \zeta_{2}(V)=-\frac{4 \pi}{\epsilon} \int_{V}^{E_{0}} \phi\left(1-\frac{E}{E_{0}}\right)\left(\frac{E^{2}}{V^{2}}-1\right)^{-\frac{1}{2}}\left(\frac{E E_{0}}{(E+V) V}-1\right) \mathrm{d} E .
\end{aligned}
$$

Consider the last term in the integral of $\zeta_{2}$. We notice that

$$
\frac{E E_{0}}{(E+V) V}-1=-\frac{1}{2}+\Gamma(\epsilon)
$$

where $\epsilon=E_{0}-V$ and $\Gamma(\epsilon)$ is a positive continuous function that satisfies $\Gamma(\epsilon) \rightarrow 0$, as $\epsilon \rightarrow 0$. So we can write for $\epsilon$ sufficiently small

$$
\left|\zeta_{2}(V)\right| \leq \frac{V}{2 \epsilon} \xi_{-\frac{1}{2}}(V)
$$

Next we consider $\zeta_{1}(V)$. We perform a change of variables, given by

$$
\begin{aligned}
\alpha & =\frac{E_{0}-E}{E_{0}}, \quad \Leftrightarrow \quad E=E_{0}(1-\alpha), \\
\mathrm{d} E & =-E_{0} \mathrm{~d} \alpha, \\
\alpha(V) & =\frac{\epsilon}{E_{0}}, \quad \alpha\left(E_{0}\right)=0 .
\end{aligned}
$$

This yields

$$
\zeta_{1}(V)=4 \pi \frac{V E_{0}}{\epsilon} \int_{0}^{\epsilon / E_{0}} \phi^{\prime}(\alpha) \alpha\left(E_{0}^{2}(1-\alpha)^{2}-V^{2}\right)^{-\frac{1}{2}} \mathrm{~d} \alpha
$$

and

$$
\xi_{-\frac{1}{2}}(V)=4 \pi E_{0} \int_{0}^{\epsilon / E_{0}} \phi(\alpha)\left(E_{0}^{2}(1-\alpha)^{2}-V^{2}\right)^{-\frac{1}{2}} \mathrm{~d} \alpha
$$

Recall that we assume that $\phi$ is analytic on $[0,1]$ and fulfills (4.6) for $n \geq 0$. This means $n$ is the lowest number such that $\phi^{(n)}(0) \neq 0\left(\phi^{(n)}\right.$ denotes the $n$-th derivative $)$. Then, we can write for all $\alpha \in[0,1]$

$$
\begin{aligned}
\phi(\alpha) & =\frac{\phi^{(n)}(0)}{n !} \alpha^{n}+\frac{\phi^{(n+1)}\left(x_{\alpha}^{1}\right)}{(n+1) !} \alpha^{n+1}, \\
\phi^{\prime}(\alpha) & =\frac{\phi^{(n)}(0)}{(n-1) !} \alpha^{n-1}+\frac{\phi^{(n+1)}\left(x_{\alpha}^{2}\right)}{n !} \alpha^{n} .
\end{aligned}
$$


where $x_{\alpha}^{1}, x_{\alpha}^{2} \in[0,1]$ in the remainder terms depend on $\alpha$. We have

$$
\begin{aligned}
& \frac{\epsilon}{4 \pi V E_{0}} \zeta_{1}(V)-\frac{1}{4 \pi E_{0}} \frac{103 n}{102} \xi_{-\frac{1}{2}}(V) \\
& =\int_{0}^{\epsilon / E_{0}}\left(\phi^{\prime}(\alpha) \alpha-\frac{103 n}{102} \phi(\alpha)\right)\left(E_{0}^{2}(1-\alpha)^{2}-V^{2}\right)^{-\frac{1}{2}} \mathrm{~d} \alpha .
\end{aligned}
$$

We consider $\left(\phi^{\prime}(\alpha) \alpha-\frac{103 n}{102} \phi(\alpha)\right)$ separately, using (4.47)-(4.48). We have for some $x_{\alpha}^{3} \in[0,1]$

$$
\begin{aligned}
\phi^{\prime}(\alpha) \alpha-\frac{103 n}{102} \phi(\alpha) & =-\frac{\alpha^{n}}{102}\left(\frac{\phi^{(n)}(0)}{(n-1) !}+\alpha \frac{\phi^{(n+1)}\left(x_{\alpha}^{3}\right)}{n !}\right) \\
& \leq \frac{\alpha^{n}}{102}\left(\epsilon \frac{\left\|\phi^{(n+1)}\right\|_{L^{\infty}([0,1])}}{n !}-\frac{\phi^{(n)}(0)}{(n-1) !}\right) .
\end{aligned}
$$

This is negative for $\epsilon$ sufficiently small. We deduce that for $\epsilon$ sufficiently small

$$
\frac{\epsilon}{4 \pi V E_{0}} \zeta_{1}(V)-\frac{1}{4 \pi E_{0}} \frac{103 n}{102} \xi_{-\frac{1}{2}}(V)<0 \Leftrightarrow \frac{\zeta_{1}(V)}{\xi_{-\frac{1}{2}}(V)}<\frac{103 n V}{102 \epsilon}
$$

Combining (4.10) from Lemma 1, (4.43) and (4.52) we obtain

$$
\left|\frac{\xi_{\frac{1}{2}}}{\xi_{-\frac{1}{2}}} \frac{\zeta}{\xi_{-\frac{1}{2}}}\right| \leq \frac{101\left(E_{0}+V\right) \epsilon}{100(3+2 n) V^{2}}\left(\frac{V}{2 \epsilon}+\frac{103 n V}{102 \epsilon}\right) .
$$

We have $E_{0}+V=2 V+\epsilon$ and if $\epsilon$ is small enough, we can write $E_{0}+V<\frac{102}{101} 2 V$. Then,

$$
\left|\frac{\xi_{\frac{1}{2}}}{\xi_{-\frac{1}{2}}} \frac{\zeta}{\xi_{-\frac{1}{2}}}\right|<\frac{102+206 n}{100(3+2 n)} \leq \frac{4}{5} .
$$

The last step is obtained by substituting $n=3$.

Proof of Theorem 2 The proof of Theorem 2 is an application of Theorem 1, the main theorem in [8]. We show that a fluid model coming from an isotropic static solution of the Einstein-Vlasov system meets the assumptions of the main theorem in [8]. In particular we will show that there exists $p_{0}>0$ such that $I(p) \leq 0$ for all $p \leq p_{0}$.

We use (4.8) and (4.31)-(4.32) to calculate

$$
\frac{\mathrm{d} \varrho}{\mathrm{d} p}=\frac{\varrho^{\prime}}{p^{\prime}}=3\left(1+\frac{2 \xi_{\frac{1}{2}}+\xi_{-\frac{1}{2}}}{4 \xi_{\frac{3}{2}}+3 \xi_{\frac{1}{2}}}\right)
$$




$$
\kappa=\frac{4 \xi_{\frac{3}{2}}+5 \xi_{\frac{1}{2}}+\xi_{-\frac{1}{2}}}{2 \xi_{\frac{3}{2}}+\xi_{\frac{1}{2}}} .
$$

A prime denotes the derivative with respect to $V$. A straightforward calculation using Lemma 3 then yields

$$
\begin{aligned}
\frac{\mathrm{d} \kappa}{\mathrm{d} p}= & \frac{\kappa^{\prime}}{p^{\prime}}=\frac{-3}{\left(2 \xi_{\frac{3}{2}}+\xi_{\frac{1}{2}}\right)^{2}\left(4 \xi_{\frac{3}{2}}+3 \xi_{\frac{1}{2}}\right)} \\
& \times\left(12 \xi_{\frac{3}{2}} \xi_{\frac{1}{2}}+18 \xi_{\frac{1}{2}}^{2}+2 \xi_{\frac{3}{2}} \xi_{-\frac{1}{2}}+8 \xi_{\frac{1}{2}} \xi_{-\frac{1}{2}}+\xi_{-\frac{1}{2}}^{2}+\left(2 \xi_{\frac{3}{2}}+\xi_{\frac{1}{2}}\right) V \xi_{-\frac{1}{2}}^{\prime}\right) .
\end{aligned}
$$

Using the formula (4.34) for $\xi_{-\frac{1}{2}}^{\prime}$ this becomes

$$
\begin{aligned}
\frac{\mathrm{d} \kappa}{\mathrm{d} p}=\frac{\kappa^{\prime}}{p^{\prime}}= & \frac{-3}{\left(2 \xi_{\frac{3}{2}}+\xi_{\frac{1}{2}}\right)^{2}\left(4 \xi_{\frac{3}{2}}+3 \xi_{\frac{1}{2}}\right)} \\
& \times\left(12 \xi_{\frac{3}{2}} \xi_{\frac{1}{2}}+18 \xi_{\frac{1}{2}}^{2}+7 \xi_{\frac{1}{2}} \xi_{-\frac{1}{2}}+\xi_{-\frac{1}{2}}^{2}-\left(2 \xi_{\frac{3}{2}}+\xi_{\frac{1}{2}}\right) \zeta\right) .
\end{aligned}
$$

Again by virtue of Lemma 1, and formula (4.56), there exists a function $\Gamma=\Gamma(V)$ which goes to zero as $V \rightarrow E_{0}$ such that we can write

$$
\begin{aligned}
\frac{1}{5} \kappa^{2} & =\frac{1}{5}\left(2 \xi_{\frac{3}{2}}+\xi_{\frac{1}{2}}\right)^{-2}\left((10+\Gamma) \xi_{\frac{1}{2}} \xi_{-\frac{1}{2}}+\xi_{-\frac{1}{2}}^{2}\right) \\
2 \kappa & =2\left(2 \xi_{\frac{3}{2}}+\xi_{\frac{1}{2}}\right)^{-2}\left[\left(2 \xi_{\frac{3}{2}}+\xi_{\frac{1}{2}}\right)\left(4 \xi_{\frac{3}{2}}+5 \xi_{\frac{1}{2}}+\xi_{-\frac{1}{2}}\right)\right] \\
& =2\left(2 \xi_{\frac{3}{2}}+\xi_{\frac{1}{2}}\right)^{-2}(1+\Gamma) \xi_{\frac{1}{2}} \xi_{-\frac{1}{2}}
\end{aligned}
$$

for $V$ close to $E_{0}$. Inserting (4.59) and (4.60) into the formula (2.3) for $I$ we derive

$$
\begin{aligned}
I= & -\left(2 \xi_{\frac{3}{2}}+\xi_{\frac{1}{2}}\right)^{-2} \\
& \times\left(\left(12 \xi_{\frac{3}{2}} \xi_{\frac{1}{2}}+18 \xi_{\frac{1}{2}}^{2}+(3-\Gamma) \xi_{\frac{1}{2}} \xi_{-\frac{1}{2}}+\frac{4}{5} \xi_{-\frac{1}{2}}^{2}-\left(2 \xi_{\frac{3}{2}}+\xi_{\frac{1}{2}}\right) \zeta\right),\right.
\end{aligned}
$$

where $\Gamma$ is a different function than that in (4.59) and (4.60), but still with the property that $\Gamma \rightarrow 0$, as $V \rightarrow E_{0}$. Now, by Lemma 4 , and since $2 \xi_{\frac{3}{2}}$ can be neglected compared to $\xi_{\frac{1}{2}}$ for $V$ close to $E_{0}$, the combination

$$
\frac{4}{5} \xi_{-\frac{1}{2}}^{2}-\left(2 \xi_{\frac{3}{2}}+\xi_{\frac{1}{2}}\right) \zeta
$$


is positive for $V$ sufficiently close to $E_{0}$. Thus, we can write

$$
I \leq-\left(2 \xi_{\frac{3}{2}}+\xi_{\frac{1}{2}}\right)^{-2}\left(\left(12 \xi_{\frac{3}{2}} \xi_{\frac{1}{2}}+18 \xi_{\frac{1}{2}}^{2}+(3-\Gamma) \xi_{\frac{1}{2}} \xi_{-\frac{1}{2}}\right)\right.
$$

By virtue of (4.9) in Lemma 1 we see that the last term,

$$
-\frac{(3-\Gamma) \xi_{\frac{1}{2}} \xi_{-\frac{1}{2}}}{\left(2 \xi_{\frac{3}{2}}+\xi_{\frac{1}{2}}\right)^{2}} \rightarrow-\infty, \quad \text { as } V \rightarrow E_{0}
$$

Since all terms in (4.63) are negative, we have obtained $I(V) \rightarrow-\infty$, as $V \rightarrow E_{0}$. Thus, by continuity there exists $V_{0}$ such that $I(V) \leq 0$ for all $V \geq V_{0}$. We set $p_{0}:=p\left(V_{0}\right)$.

\section{Discussion}

\subsection{Limits of the Einstein-Vlasov system}

In the last section we showed that if a static, isotropic solution of the Einstein-Vlasov system does not have too high pressure, it is the unique spherically symmetric solution to the prescribed ansatz function $\phi$ and surface potential $E_{0}$.

Now we consider different limits to get some insights when Theorem 1 can be applied and in what situations the assumptions are not met. Fist we consider situations of high pressure, i.e., the relativistic limit for massive particles, where $v^{2} \gg m^{2}$. We find with (3.4), (3.5)

$$
\rho \simeq 4 \pi \int_{0}^{\infty} v^{3} F(x, v) \mathrm{d} v, \quad p \simeq \frac{1}{3} \rho .
$$

Hence, the equation of state for radiation fluid, $\varrho(p)=3 p$, is recovered. For this equation of state $I(p)$ can easily be calculated. One obtains $I(p)=\frac{24}{5}>0$, and Theorem 1 cannot be applied.

In the non-relativistic limit for massive particles, where $v^{2} \ll m^{2}$, we find

$$
\rho \simeq N m=4 \pi \int_{0}^{\infty} v^{2} F(x, v) \mathrm{d} v, \quad p \simeq \frac{1}{3} m\left\langle v^{2}\right\rangle N
$$

where

$$
\left\langle v^{2}\right\rangle:=\frac{\int_{0}^{\infty} v^{2} \mathrm{~d} v \frac{\mathrm{d} N}{\mathrm{~d} v}}{\int_{0}^{\infty} \frac{\mathrm{d} N}{\mathrm{~d} v} \mathrm{~d} v}
$$

and $N$ is defined by $N^{\mu}=N u^{\mu}$ where $N^{\mu}$ is the particle number current defined in (2.10) and $u^{\mu}$ is the four velocity of the fluid, given in (3.12). Thus, the equation of state 
for non-relativistic ideal gas, $\varrho(p)=\frac{3}{\left\langle v^{2}\right\rangle} p$, is recovered. For this equation of state, with $\left\langle v^{2}\right\rangle$ constant, corresponding to isothermal gas, we obtain $I>0$, independent of $p$, as well.

For the massless case, where $m=0$, we can explicitly see

$$
\rho=4 \pi \int_{0}^{\infty} v^{3} F(x, v) \mathrm{d} v, \quad p=\frac{1}{3} \rho,
$$

and hence $p=\rho / 3$ is recovered. We should note that thermal equilibrium is not necessary for $p=\rho / 3$. In other words, if we assume isotropy in the momentum space in the Vlasov system of massless particles, it necessarily reduces to the perfect fluid system with the equation of state $p=\rho / 3$. We have already seen that for this equation of state one always has $I>0$, independently of $\phi$ and $E_{0}$.

Here, it is instructive to derive the equation of state in the low-pressure regime for the massive case in the context of Theorem 2. For simplicity, in reference to (4.6), we assume that $\Phi(E)=\phi\left(1-E / E_{0}\right)$ has a cut off, i.e., $\phi(x)=0$ for $x<0$ and $\lim _{x \rightarrow+0} x^{-n} \phi(x)=C>0$ for $n \geq 0$, where $n$ is not necessarily integer here. If $V<E_{0}$ and $V$ is sufficiently close to $E_{0},(4.4)$ and (4.5) yield

$$
\begin{aligned}
& \rho \approx 4 \sqrt{2} \pi C \epsilon^{n+3 / 2} A_{n}, \\
& p \approx 2^{3 / 2} \frac{4}{3} \pi C \epsilon^{n+5 / 2} B_{n},
\end{aligned}
$$

in the lowest order, where

$$
A_{n}=\frac{\sqrt{\pi} \Gamma(n+1)}{2 \Gamma(n+5 / 2)}, \quad B_{n}=\frac{3 \sqrt{\pi} \Gamma(n+1)}{4 \Gamma(n+7 / 2)}, \quad \epsilon=\frac{E_{0}}{V}-1 .
$$

Thus, we obtain the following polytropic equation of state

$$
p \approx K \rho^{\gamma}
$$

where

$$
\gamma=\frac{2 n+5}{2 n+3}, \quad K=\frac{2}{3}[4 \sqrt{2} \pi C]^{-2 /(2 n+3)} \frac{B}{A^{(2 n+5) /(2 n+3)}}
$$

or $\rho=\tilde{K} p^{\gamma^{-1}}$ with $\tilde{K}=K^{-\gamma^{-1}}$. In this lowest order, we can easily find

$$
I \approx-\frac{5 \gamma-6}{5 \gamma^{2}} \tilde{K}^{2} p^{2(1-\gamma) / \gamma}
$$

Therefore, if $V$ is sufficiently close to $E_{0}$, or equivalently, $p$ is sufficiently small, $I$ is negative and hence Theorem 1 applies for $\gamma>6 / 5$ or $0 \leq n<7 / 2$. It is interesting to note that $\gamma=6 / 5$ is the critical value beyond which the polytrope has a surface of finite radius in Newtonian gravity. Note that the equation of state for the low-pressure regime cannot be that for isothermal gas. 


\subsection{Example of a step-function energy distribution}

In this section and Sect. 5.3 we construct an explicit family of examples of static solutions of the Einstein-Vlasov system that are spherically symmetric and unique.

Let $E_{0}>0$. We consider the ansatz

$$
F(\mathbf{x}, v)=\Phi(E)=\Theta\left(E_{0}-E\right),
$$

where $\Theta$ denotes the Heaviside step function. This ansatz describes a particle distribution where the energy is evenly distributed over the particles up until a cutoff energy $E_{0}$.

We can calculate $\varrho(V)$ and $p(V)$ explicitly from the formulas (4.4) and (4.5). We obtain

$$
\begin{aligned}
& \varrho(V)=\frac{\pi E_{0}}{2 V} \sqrt{\left(\frac{E_{0}}{V}\right)^{2}-1}\left(2\left(\frac{E_{0}}{V}\right)^{2}-1\right)-\frac{\pi}{2} \ln \left(\sqrt{\left(\frac{E_{0}}{V}\right)^{2}-1}+\frac{E_{0}}{V}\right), \\
& p(V)=\frac{E_{0} \pi}{6 V} \sqrt{\left(\frac{E_{0}}{V}\right)^{2}-1}\left(2\left(\frac{E_{0}}{V}\right)^{2}-5\right)+\frac{\pi}{2} \ln \left(\sqrt{\left(\frac{E_{0}}{V}\right)^{2}-1}+\frac{E_{0}}{V}\right) .
\end{aligned}
$$

For the function $I(V)$ an explicit formula can be obtained, too. We have

$$
I=\frac{\varrho+p}{5(\varrho+3 p)^{2}}\left(30 p \frac{\mathrm{d} \varrho}{\mathrm{d} p}+(\varrho+11 p)\left(\frac{\mathrm{d} \varrho}{\mathrm{d} p}\right)^{2}+5\left(3 p^{2}+4 p \varrho+\varrho^{2}\right) \frac{\mathrm{d}^{2} \varrho}{\mathrm{d} p^{2}}\right)
$$

We calculate

$$
\begin{aligned}
\frac{\mathrm{d} \varrho}{\mathrm{d} p} & =\frac{\mathrm{d} \varrho}{\mathrm{d} V} \frac{\mathrm{d} V}{\mathrm{~d} p}=\frac{\frac{\mathrm{d} \varrho}{\mathrm{d} V}}{\frac{\mathrm{d} p}{\mathrm{~d} V}}=\frac{3 E_{0}^{2}}{E_{0}^{2}-V^{2}} \\
\frac{\mathrm{d}^{2} \varrho}{\mathrm{d} p^{2}} & =\frac{1}{\left(\frac{\mathrm{d} p}{\mathrm{~d} V}\right)^{3}}\left(\frac{\mathrm{d}^{2} \varrho}{\mathrm{d} V^{2}} \frac{\mathrm{d} p}{\mathrm{~d} V}-\frac{\mathrm{d} \varrho}{\mathrm{d} V} \frac{\mathrm{d}^{2} p}{\mathrm{~d} V^{2}}\right)=-\frac{9 E_{0} V^{7}}{2 \pi\left(E_{0}^{2}-V^{2}\right)^{4}} \sqrt{\frac{E_{0}^{2}}{V^{2}}-1}
\end{aligned}
$$

Inserting into (5.14) yields

$$
I(V)=4 E_{0}^{3}\left(24 E_{0}^{7}-108 E_{0}^{5} V^{2}+139 E_{0}^{3} V^{4}-55 E_{0} V^{6}\right.
$$




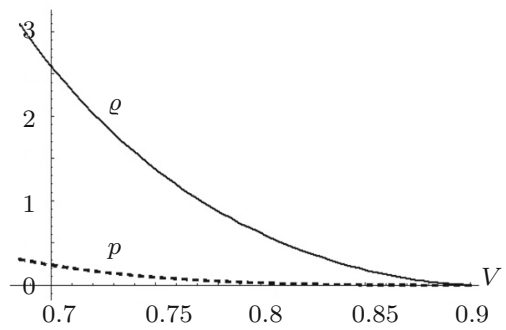

Fig. $1 \varrho(V)$ and $p(V)$ (dashed) for $E_{0}=0.9$

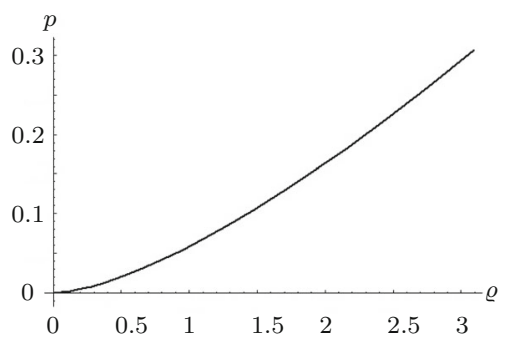

Fig. 2 Equation of state $p(\varrho)$ for the step function ansatz (5.11) and $E_{0}=0.9$

$$
\begin{aligned}
& \left.-5 \sqrt{\frac{E_{0}^{2}}{V^{2}}-1 V^{5}}\left(5 V^{2}-6 E_{0}^{2}\right) \ln \left(\sqrt{\frac{E_{0}^{2}}{V^{2}}-1}+\frac{E_{0}}{V}\right)\right) \\
& \times\left(5 ( E _ { 0 } ^ { 2 } - V ^ { 2 } ) \left(E_{0} \sqrt{\frac{E_{0}^{2}}{V^{2}}-1} V\left(2 E_{0}^{2}-3 V^{2}\right)\right.\right. \\
& \left.\left.+V^{4} \ln \left(\sqrt{\frac{E_{0}^{2}}{V^{2}}-1}+\frac{E_{0}}{V}\right)\right)^{2}\right)^{-1} .
\end{aligned}
$$

Using L'Hôpital's rule we can confirm that $I(V) \rightarrow-\infty$, as $V \rightarrow E_{0}$, which corresponds to $p \rightarrow 0$ (as stated in Theorem 2). If a particular choice for $E_{0}$ is made a certain equation of state is fixed and one can calculate the corresponding pressure $p_{0}$ such that $I(p) \leq 0$ for all $p \leq p_{0}$ by solving the equation $I\left(V\left(p_{0}\right)\right)=0$. To illustrate this we consider the example $E_{0}=0.9$. The resulting functions for $\varrho(V)$ and $p(V)$ can be found in Fig. 1. The equation of state is illustrated in Fig. 2. Finally we obtain $I(V)$ as drawn in Fig. 3. We calculate $V_{0} \approx 0.68508$ and the corresponding $p_{0}=p\left(V_{0}\right) \approx 0.30645$. As shown in Sect. 4 , a spherically symmetric static solution of the Einstein-Vlasov system with the ansatz (5.11) is unique, if $\sup _{x} p(x) \leq p_{0}$. However, at this stage of the analysis it is still open if there exist static solutions corresponding to the ansatz (5.11) with cutoff energy $E_{0}=0.9$ which satisfy $\sup _{x \in \mathbb{R}^{3}} p(x) \leq p_{0}$. This issue is addressed in the next section. 


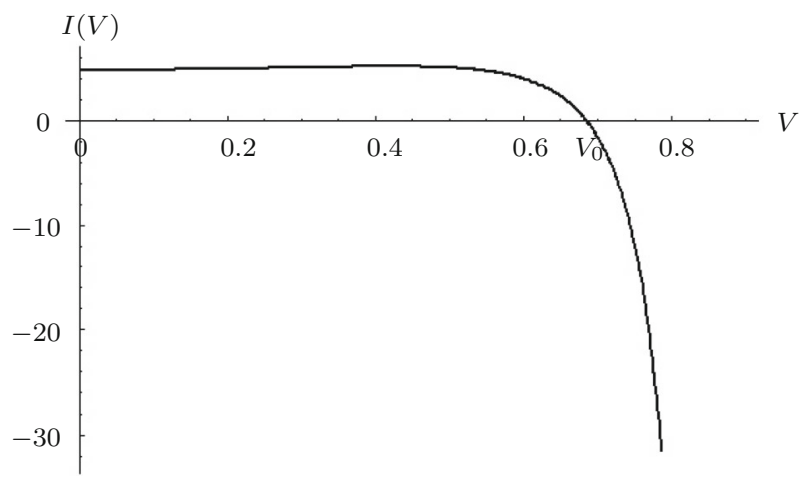

Fig. 3 Plot of $I(V)$ for $E_{0}=0.9$

\subsection{Parameters for unique static solutions}

Now we construct static solutions of the spherically symmetric Einstein-Vlasov system by numerical means to obtain some insights into the condition that $\sup _{x} p(x)$ must not be too large. For a spherically symmetric regular solution, the space-time manifold is $\mathscr{M} \cong \mathbb{R} \times \mathbb{R}^{3}$ and can be equipped with coordinates $t \in \mathbb{R}, r \in[0, \infty), \vartheta \in$ $[0, \pi], \varphi \in[0,2 \pi)$ such that the metric reads

$$
g=-e^{2 \mu(r)} \mathrm{d} t^{2}+e^{2 \lambda(r)} \mathrm{d} r^{2}+r^{2} \mathrm{~d} \vartheta^{2}+r^{2} \sin ^{2} \vartheta \mathrm{d} \varphi^{2}
$$

for functions $\mu, \lambda: \mathbb{R}_{+} \rightarrow \mathbb{R}$. Define the Hawking mass

$$
m(r)=4 \pi \int_{0}^{r} s^{2} \varrho(s) \mathrm{d} s .
$$

It can be shown that in spherical symmetry the static Einstein-Vlasov system reduces to the integro-differential equation

$$
\begin{aligned}
\mu^{\prime}(r) & =\frac{1}{1-\frac{2 m(r)}{r}}\left(4 \pi r p(r)+\frac{m(r)}{r^{2}}\right), \\
\mu(0) & =\mu_{c}<0,
\end{aligned}
$$

cf. [15]. We observe that $V(r)=e^{\mu(r)}$ is strictly increasing. Since $p(V)$ is decreasing in $V$, cf. 4.5), the maximum value will be attained at $V_{c}:=e^{\mu_{c}}$. So for a spherically symmetric solution we have

$$
\sup _{x \in \mathbb{R}^{3}} p(x)=p\left(e^{\mu_{c}}\right) .
$$

The aim now is to find values for $E_{0}$ and $\mu_{c}$ such that the corresponding spherically symmetric solution satisfies $\sup _{x} p(x) \leq p_{0}$ and thus is unique. 
A spherically symmetric static solution of the Einstein-Vlasov system can be calculated numerically by integrating (5.20a)-(5.20b) using the methods described in [5]. Thereby, a solution is (uniquely) determined by the choice of $\mu_{c}$ and $E_{0}$. When integrating the function $\mu(r)$ outwards along the radial axis, it will asymptotically approach a fixed value

$$
\mu_{\infty}:=\lim _{r \rightarrow \infty} \mu(r)
$$

An asymptotically flat solution, however, satisfies $\mu_{\infty}=0$. After a numerical solution has been constructed, this can be achieved by a rescaling of the time coordinate. Since $E=g\left(\partial_{t}, p\right)$, this in turn affects the particle energy and changes the role of the cutoff energy $E_{0}$. So $\mu_{\infty}, \mu_{c}$, and $E_{0}$ are not independent parameters and the construction of asymptotically flat, spherically symmetric static solutions requires a bit more care.

As described, for example, in [5], it can be done by introducing the variable $y=$ $e^{\mu} / E_{0}$. This substitution makes the cutoff energy $E_{0}$ disappear as a free parameter from the problem, as can be seen as follows. Using the formula (4.1) for the energy $E$, we obtain

$$
\Phi(E)=\Theta\left(E_{0}-E\right)=\left[1-\frac{e^{\mu} \sqrt{1+v^{2}}}{E_{0}}\right]_{+}=\left[1-y \sqrt{1+v^{2}}\right]_{+} .
$$

The system (5.20a)-(5.20b) becomes

$$
\begin{aligned}
y^{\prime}(r) & =\frac{y(r)}{1-\frac{2 m(r)}{r}}\left(4 \pi r p(r)+\frac{m(r)}{r^{2}}\right), \\
y(0) & =y_{c}<1 .
\end{aligned}
$$

A solution in terms of $y$ is then uniquely (as a spherically symmetric solution) determined by the central value $y_{c}:=y(r=0)$. After a solution in terms of $y$ has been constructed, the values of $\mu_{c}$ and $E_{0}$ can be calculated, such that $\mu_{\infty}=0$.

In this context we would like to construct a solution with minimal potential $V_{c}:=$ $e^{\mu_{c}}$ fulfilling $V_{0} \leq V_{c}$, where $V_{0}$ is the zero of $I(V)$, which is solely determined by the choice of $E_{0}$ (and $\left.\phi\right)$. For such a solution we have $p(x) \leq p\left(e^{\mu_{c}}\right)$ and $I(V) \leq 0$ everywhere.

Since, as just described, the values for $\mu_{c}$ and $E_{0}$ cannot be chosen directly, we carry out a parameter study, cf. Fig. 4 . First, for each value of $E_{0}$, we calculate $V_{0}$ by solving $I\left(V_{0}\right)=0$, where $I(V)$ is given in (5.17). We obtain the dashed line. Then, we calculate steady states for a succession of central values $y_{c}$ between 0.1 and 0.99 , by integrating $(5.24 \mathrm{a})-(5.24 \mathrm{~b})$. Linking the $\left(E_{0}, e^{\mu_{c}}\right)$-points corresponding to these solutions one obtains the continuous line in Fig. 4. The part of the continuous line lying above the dashed line corresponds to solutions with $V_{0} \leq e^{\mu_{c}}$, lying in the lowpressure regime. The intersection (marked with a " $\oplus$ " in Fig. 4) lies approximately at $\hat{y}_{c}=0.76$, corresponding to $\hat{E}_{0} \approx 0.875, \hat{V}_{c} \approx 0.665$. In order to compare with the example, depicted in Figs. 1 and 2, we consider a solution corresponding to $y_{c}=0.83$, $E_{0} \approx 0.902, V_{c} \approx 0.748$ (marked with an " $\otimes$ " in Fig. 4). If we compare this value 


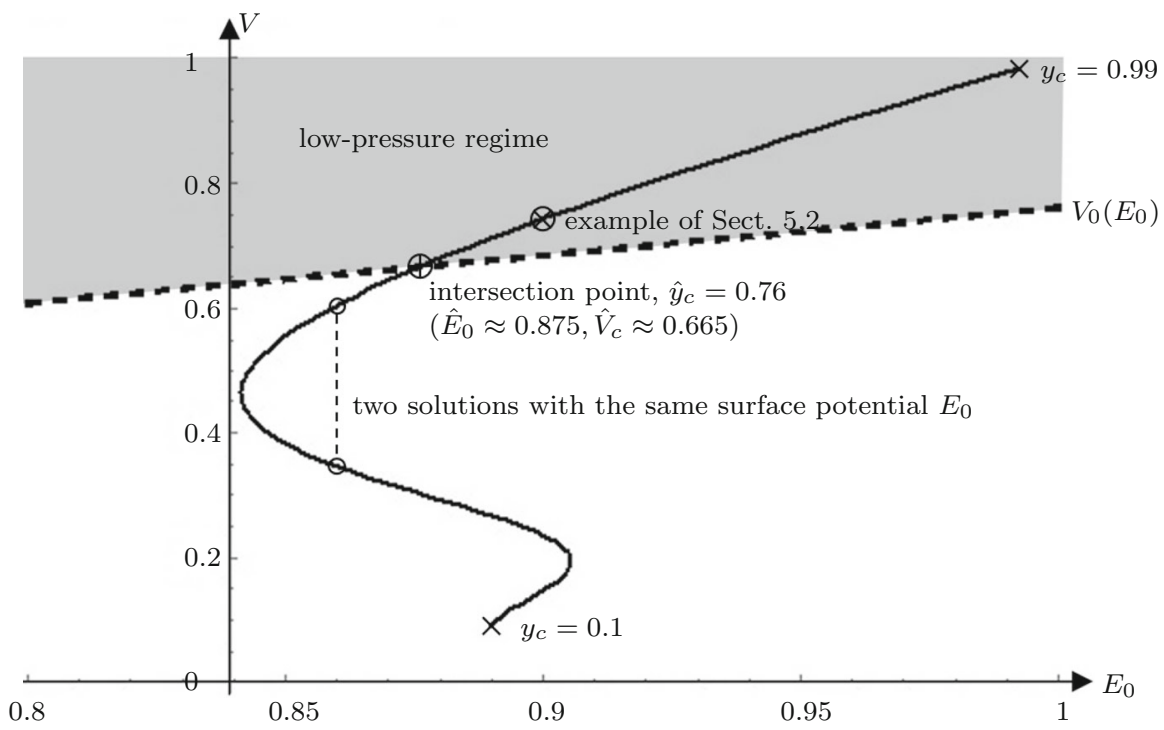

Fig. 4 For each choice of $E_{0}$ the function $I(V)$ has one zero which is called $V_{0}\left(E_{0}\right)$. The tuples $\left(E_{0}, V_{0}\right)$ lie on the dashed line. The continuous line represents a succession of central values $y_{c}$ for $y(r)$ between 0.1 and 0.99 (marked with $\times$ ), and the resulting tuples $\left(E_{0}, V_{c}\right)$, where $V_{c}=e^{\mu_{c}}$ is the (minimal) potential at the center. The solution at the intersection is marked with $\oplus$ and denoted with a hat. The solution which has been discussed as an example in Sects. 5.2 and 5.3 above is marked with a $\otimes$

for $V_{c}$ to $V_{0} \approx 0.685$ that we have calculated above for $E_{0}=0.9$, we see that this particular spherically symmetric solution is in the realm where the uniqueness theorem (Theorem 2) is valid.

Figure 4 contains two messages: first, that there indeed exist static solutions of the Einstein-Vlasov system in the low-pressure regime, i.e., solutions that the uniqueness theorem applies to. Second, it gives some indication that uniqueness does not hold for arbitrary pressures. Observe that the continuous line takes no turns above the dashed line (in the low-pressure regime), whereas it oscillates below the dashed line. If the continuous line oscillates, this means that there are several spherically symmetric solutions corresponding to the same surface potential $E_{0}$. One example $\left(E_{0} \approx 0.86\right)$ is marked by "o" in Fig. 4. So in the realm below the dashed line the solutions are not unique, not even under the restriction of spherical symmetry. The question whether or not there also exist several solutions with the same surface potential, which are not spherically symmetric, can of course not be answered with this parameters study. Furthermore, for some solutions in the low-pressure regime there exist solutions with higher pressures that have the same surface potential. For example, for $E_{0} \approx 0.902$, we can see that there are two solutions below the dashed line with $y_{c}$ between 0.1 and 0.76 besides the unique solution marked with an " $\otimes$ " above the dashed line. So, in conclusion, uniqueness seems to hold only within the low-pressure regime. In other words, the uniqueness theorem in the current paper assures that for a given value of surface potential, the low-pressure static solution in an asymptotically flat space-time is spherically symmetric and uniquely determined, without excluding the existence of high-pressure solutions which may be or may not be spherically symmetric. 


\subsection{Comparison to astrophysical objects}

The concentration parameter

$$
\Gamma=\sup _{r \in(0, \infty)} \frac{2 m(r)}{r}
$$

is a dimensionless quantity indicating how relativistic the solution is. The solution is very relativistic if $\Gamma$ is large. As a generalization to the well-known Buchdahl inequality, the upper bound $\Gamma \leq \frac{8}{9}$ has been shown for a large class of matter models including Vlasov matter and the perfect fluid model [2]. Moreover, the smaller $\mu_{c}<0$ is chosen (or equivalently the smaller $y_{c}$ is chosen, cf. Fig. 4) the bigger the value of $\Gamma$ will be. This has been made precise in [3].

Let $\hat{\Gamma}$ be the concentration parameter of the solution corresponding to $\hat{y}_{c}$, the intersection point in Fig. 4. Then, if another solution has $\Gamma<\hat{\Gamma}$, its maximal (central) potential $V_{c}$ will be larger than the maximal potential $\hat{V}_{c}$ of the critical solution. This means it will be unique. We calculate $\hat{\Gamma} \approx 0.292$. It might be instructive to set this number into relation to values of $\Gamma$ for other objects in the universe.

Taking into account physical units, at the surface of a spherical object with mass $M$ and radius $R$ we have $\Gamma=2 M G /\left(c^{2} r\right)$, where $G$ is the gravitational constant and $c$ is the speed of light. A very extreme situation is the surface of a neutron star. The survey article [13] suggests that for a model calculation one can assume $M=3 \cdot 10^{30} \mathrm{~kg}$ and $R=10 \mathrm{~km}$. This yields $\Gamma \approx 0.4424$. A value clearly larger than $\hat{\Gamma}$ for the family with ansatz function given in (5.11). So Theorem 1 is not suitable in the regime of strong gravity like at the surface of a neutron star. The authors of [8], where Theorem 1 emanates from, make a similar remark.

Taking radius and mass of the sun however, we calculate $\Gamma \approx 4.24 \cdot 10^{-6}$ at the surface of the sun. This is clearly smaller than $\hat{\Gamma}$. As application of Theorem 2 we have globular clusters in mind. Since the typical mass and size of a globular cluster are hundreds of thousands solar masses and several parsecs, respectively, the ratio $\Gamma$ is approximately $10^{-8}$. This means that if the distribution function is a decreasing function of $E$ up to the cut off $E_{0}<\infty$, Theorem 1 applies to globular clusters.

Finally we remark that there might be a connection between stability and the fact that a solution necessarily is spherically symmetric, like in the non-relativistic case. Even though only very little is known about the question of stability of static solutions of the Einstein-Vlasov system, it is conjectured that solutions with small values of $\Gamma$ are stable, whereas highly relativistic solutions with large values of $\Gamma$ are conjectured to be unstable. The reader is referred to [6] for a numerical study of this question.

Acknowledgements Open access funding provided by Chalmers University of Technology. We thank Jérémie Joudioux for mentioning [8] to us, even though he might have had completely different questions in mind. T.H. is grateful to T. Shiromizu for fruitful discussion. M.T. thanks Håkan Andréasson and Simone Calogero for helpful discussions. Moreover, M.T. is grateful to the Department of Theoretical Physics at Rikkyo University for hospitality during a research stay June-August 2017, as well as the Japan Society for the Promotion of Science (JSPS) and the Swedish Foundation for International Cooperation in Research and Higher Education (STINT) for financial support. This work was partially supported by JSPS KAKENHI Grant No. JP26400282 (T.H.). 


\section{Compliance with ethical standards}

Conflict of interest On behalf of all authors, the corresponding author states that there is no conflict of interest.

Open Access This article is licensed under a Creative Commons Attribution 4.0 International License, which permits use, sharing, adaptation, distribution and reproduction in any medium or format, as long as you give appropriate credit to the original author(s) and the source, provide a link to the Creative Commons licence, and indicate if changes were made. The images or other third party material in this article are included in the article's Creative Commons licence, unless indicated otherwise in a credit line to the material. If material is not included in the article's Creative Commons licence and your intended use is not permitted by statutory regulation or exceeds the permitted use, you will need to obtain permission directly from the copyright holder. To view a copy of this licence, visit http://creativecommons.org/licenses/by/4.0/.

\section{References}

1. Andréasson, H.: The Einstein-Vlasov system/kinetic theory. Living Rev. Relativ. 14, 4 (2011)

2. Andréasson, H.: Sharp bounds on $2 \mathrm{~m} / \mathrm{r}$ of general spherically symmetric static objects. J. Differ. Equ. 245, 2243-2266 (2009)

3. Andréasson, H.: On static shells and the Buchdahl inequality for the spherically symmetric EinsteinVlasov system. Commun. Math. Phys. 274, 409-425 (2007)

4. Andréasson, H., Kunze, M., Rein, G.: Existence of axially symmetric static solutions of the EinsteinVlasov system. Commun. Math. Phys. 308, 23-47 (2011)

5. Andréasson, H., Rein, G.: On the steady states of the spherically symmetric Einstein-Vlasov system. Class. Quantum Grav. 24, 1809-1832 (2007)

6. Andréasson, H., Rein, G.: A numerical investigation of the stability of steady states and critical phenomena for the spherically symmetric Einstein-Vlasov system. Class. Quantum Grav. 23, 3659-3677 (2006)

7. Batt, J., Faltenbacher, W., Horst, E.: Stationary spherically symmetric models in stellar dynamics. Arch. Ration. Mech. Anal. 93, 159-183 (1986)

8. Beig, R., Simon, W.: On the uniqueness of static perfect-fluid solutions in general relativity. Commun. Math. Phys. 144, 373-390 (1992)

9. Hadzic, M., Rein, G.: Stability for the spherically symmetric Einstein-Vlasov system: a coercivity estimate. arXiv:1205.6198

10. Lichtenstein, L.: Gleichgewichtsfiguren rotierender Flüssigkeiten. Springer, Berlin (1933)

11. Lindblom, L.: On the symmetries of equilibrium stellar models. Philos. Trans. Phys. Sci. Eng. 340(1658), 353-364 (1992)

12. Masood-ul-Alam, A.K.M.: A proof of uniqueness of static stellar models with small $d \varrho / d p$. Class. Quantum Grav. 5, 409-421 (1988)

13. Özel, F., Freire, P.: Massess, Radii, and Equation of State of Neutron Stars. arXiv:1603.02698v1

14. Rein, G., Rendall, A.: Smooth static solutions of the spherically symmetric Vlasov-Einstein system. Ann. Inst. Henri Poincaré A 59, 383-397 (1993)

15. Rein, G., Rendall, A.: Global existence of solutions of the spherically symmetric Vlasov-Einstein system. Commun. Math. Phys. 150, 585-591 (1991)

16. Rein, G.: Collisionless Kinetic Equations from Astrophysics: The Vlasov-Poisson System

17. Rein, G., Rendall, A.: Compact support of spherically symmetric equilibria in non-relativistic and relativisticgalactic dynamics. Math. Proc. Camb. Phil. Soc. 128, 363-380 (2000)

18. Sarbach, O., Zannias, T.: The geometry of the tangent bundle and the relativistic kinetic theory of gases. Class. Quant. Grav. 31, 085013 (2014)

Publisher's Note Springer Nature remains neutral with regard to jurisdictional claims in published maps and institutional affiliations. 